\title{
Compositions and convex combinations of asymptotically regular firmly nonexpansive mappings are also asymptotically regular
}

\author{
Heinz H Bauschke ${ }^{1 *}$, Victoria Martín-Márquez ${ }^{2}$, Sarah M Moffat ${ }^{1}$ and Xianfu Wang ${ }^{1}$
}

\author{
* Correspondence: heinz. \\ bauschke@ubc.ca \\ ${ }^{1}$ Mathematics, University of British \\ Columbia, Kelowna, BC V1V 1V7, \\ Canada \\ Full list of author information is \\ available at the end of the article
}

\begin{abstract}
Because of Minty's classical correspondence between firmly nonexpansive mappings and maximally monotone operators, the notion of a firmly nonexpansive mapping has proven to be of basic importance in fixed point theory, monotone operator theory, and convex optimization. In this note, we show that if finitely many firmly nonexpansive mappings defined on a real Hilbert space are given and each of these mappings is asymptotically regular, which is equivalent to saying that they have or "almost have" fixed points, then the same is true for their composition. This significantly generalizes the result by Bauschke from 2003 for the case of projectors (nearest point mappings). The proof resides in a Hilbert product space and it relies upon the Brezis-Haraux range approximation result. By working in a suitably scaled Hilbert product space, we also establish the asymptotic regularity of convex combinations.
\end{abstract}

2010 Mathematics Subject Classification: Primary 47H05, 47H09; Secondary 47H10, 90C25.

Keywords: asymptotic regularity, firmly nonexpansive mapping, Hilbert space, maximally monotone operator, nonexpansive mapping, resolvent, strongly nonexpansive mapping

\section{Introduction and standing assumptions}

Throughout this article,

$X$ is a real Hilbert space with inner product $\langle\cdot, \cdot\rangle$

and induced norm $\|\cdot\|$. We assume that

$$
m \in\{2,3,4, \ldots\} \text { and } I:=\{1,2, \ldots, m\} .
$$

Recall that an operator $T: X \rightarrow X$ is firmly nonexpansive (see, e.g., [1-3] for further information) if $(\forall x \in X)(\forall y \in X)\|T x-T y\|^{2} \leq\langle x-y, T x-T y\rangle$ and that a set-valued operator $A$ : $X \rightrightarrows X$ is maximally monotone if it is monotone, i.e., for all $\left(x, x^{*}\right)$ and $(y$, $\left.y^{*}\right)$ in the graph of $A$, we have $\left\langle x-y, x^{*}-y^{*}\right\rangle \geq 0$ and if the graph of $A$ cannot be properly enlarged without destroying monotonicity (We shall write $\operatorname{dom} A=\{x \in X \mid A x \neq$ $\varnothing\}$ for the domain of $A, \operatorname{ran} A=A(X)=\cup_{x \in X} A x$ for the range of $A$, and gr $A$ for the graph of $A$.) These notions are equivalent (see [4,5]) in the sense that if $A$ is maximally monotone, then its resolvent $J_{A}:=(\mathrm{Id}+A)^{-1}$ is firmly nonexpansive, and if $T$ is firmly

(c) 2012 Bauschke et al; licensee Springer. This is an Open Access article distributed under the terms of the Creative Commons Attribution License (http://creativecommons.org/licenses/by/2.0), which permits unrestricted use, distribution, and reproduction in any medium, provided the original work is properly cited. 
nonexpansive, then $T^{-1}$ - Id is maximally monotone. (Here and elsewhere, Id denotes the identity operator on $X$.) The Minty parametrization (see [4] and also [[1], Remark 23.22(ii)]) states that if $A$ is maximally monotone, then

$$
\operatorname{gr} A=\left\{\left(J_{A} x, x-J_{A} x\right) \mid x \in X\right\} .
$$

In optimization, one main problem is to find zeros of maximally monotone operators- these zeros may correspond to critical points or solutions to optimization problems. In terms of resolvents, the corresponding problem is that of finding fixed points. For background material in fixed point theory and monotone operator theory, we refer the reader to [1-3,6-16].

The aim of this note is to provide approximate fixed point results for compositions and convex combinations of finitely many firmly nonexpansive operators.

The first main result (Theorem 4.6) substantially extends a result by Bauschke [17] on the compositions of projectors to the composition of firmly nonexpansive mappings. The second main result (Theorem 5.5) extends a result by Bauschke, Moffat and Wang [18] on the convex combination of firmly nonexpansive operators from Euclidean to Hilbert space.

The remainder of this section provides the standing assumptions used throughout the article.

Even though the main results are formulated in the given Hilbert space $X$, it will turn out that the key space to work in is the product space

$$
X^{m}:=\left\{\mathbf{x}=\left(x_{i}\right)_{i \in I} \mid(\forall i \in I) x_{i} \in X\right\} .
$$

This product space contains an embedding of the original space $X$ via the diagonal subspace

$$
\boldsymbol{\Delta}:=\left\{\mathbf{x}=(x)_{i \in I} \mid x \in X\right\} .
$$

We also assume that we are given $m$ firmly nonexpansive operators $T_{1}, \ldots, T_{m}$; equivalently, $m$ resolvents of maximally monotone operators $A_{1}, \ldots, A_{m}$ :

$$
(\forall i \in I) \quad T_{i}=J_{A_{i}}=\left(\mathrm{Id}+A_{i}\right)^{-1} \text { is firmly nonexpansive. }
$$

We now define various pertinent operators acting on $X^{m}$. We start with the Cartesian product operators

$$
\text { T: } X^{m} \rightarrow X^{m}:\left(x_{i}\right)_{i \in I} \mapsto\left(T_{i} x_{i}\right)_{i \in I}
$$

and

$$
\text { A: } X^{m} \rightrightarrows X^{m}:\left(x_{i}\right)_{i \in I} \mapsto\left(A_{i} x_{i}\right)_{i \in I} .
$$

Denoting the identity on $X^{m}$ by Id, we observe that

$$
J_{\mathrm{A}}=(\mathbf{I d}+\mathbf{A})^{-1}=T_{1} \times \cdots \times T_{m}=\mathbf{T} .
$$

Of central importance will be the cyclic right-shift operator

$$
\mathbf{R}: X^{m} \rightarrow X^{m}:\left(x_{1}, x_{2} \ldots, x_{m}\right) \mapsto\left(x_{m}, x_{1}, \ldots, x_{m-1}\right)
$$

and for convenience we set

$$
M=I d-R .
$$


We also fix strictly positive convex coefficients (or weights) $\left(\lambda_{i}\right)_{i \in I}$, i.e.,

$$
\left.(\forall i \in I) \quad \lambda_{\mathrm{i}} \in\right] 0,1\left[\text { and } \quad \sum_{i \in I} \lambda_{i}=1 .\right.
$$

Let us make $X^{m}$ into the Hilbert product space

$$
\mathbf{X}:=X^{m}, \quad \text { with } \quad\langle\mathbf{x}, \mathbf{y}\rangle=\sum_{i \in I}\left\langle x_{i}, y_{i}\right\rangle .
$$

The orthogonal complement of $\Delta$ with respect to this standard inner product is known (see, e.g., [[1], Proposition 25.4(i)]) to be

$$
\boldsymbol{\Delta}^{\perp}=\left\{\mathbf{x}=\left(x_{i}\right)_{i \in I} \mid \sum_{i \in I} x_{i}=0\right\} .
$$

Finally, given a nonempty closed convex subset $C$ of $X$, the projector (nearest point mapping) onto $C$ is denoted by $P_{C}$. It is well known to be firmly nonexpansive.

\section{Properties of the operator $M$}

In this section, we collect several useful properties of the operator $\mathbf{M}$, including its Moore-Penrose inverse (see [19] and e.g., [[1], Section 3.2] for further information.). To that end, the following result-which is probably part of the folklore-will turn out to be useful.

Proposition 2.1 Let $Y$ be a real Hilbert space and let $B$ be a continuous linear operator from $X$ to $Y$ with adjoint $B^{*}$ and such that $\operatorname{ran} B$ is closed. Then the Moore-Penrose inverse of $B$ satisfies

$$
B^{\dagger}=P_{\operatorname{ran} B^{*}} \circ B^{-1} \circ P_{\operatorname{ran~} B} .
$$

Proof Take $y \in Y$. Define the corresponding set of least squares solutions (see, e.g., [[1], Proposition 3.25]) by $C:=B^{-1}\left(P_{\operatorname{ran} B} y\right)$. Since $\operatorname{ran} B$ is closed, so is $\operatorname{ran} B^{*}$ (see, e. g., [[1], Corollary 15.34]); hence, ${ }^{\mathrm{a}} U:=(\operatorname{Ker} B)^{\perp}=\overline{\operatorname{ran} B^{*}}=\operatorname{ran} B^{*}$. Thus, $C=B^{\dagger} y+$ ker $B=B^{\dagger} y+U^{\dagger}$. Therefore, since $\operatorname{ran} B^{\dagger}=\operatorname{ran} B^{*}$ (see, e.g., [[1], Proposition 3.28(v)]), $P_{U}$ $(C)=P_{U} B^{\dagger} y=B^{\dagger} y$, as claimed.

Before we present various useful properties of $\mathbf{M}$, let us recall the notion of a rectangular (which is also known as star or $3^{*}$ monotone, see [20]) operator. A monotone operator $B: \mathrm{X} \rightrightarrows \mathrm{X}$ is rectangular if $\left(\forall\left(x, y^{*}\right) \in \operatorname{dom} B \times \operatorname{ran} B\right) \sup _{\left(z, z^{*}\right) \in \operatorname{gr} B}\left\langle x-z, z^{*}-y\right\rangle<+\infty$.

Theorem 2.2 Define $^{\mathrm{b}}$

$$
\mathbf{L}: \boldsymbol{\Delta}^{\perp} \rightarrow \mathbf{X}: \mathbf{y} \mapsto \sum_{i=1}^{m-1} \frac{m-i}{m} \mathbf{R}^{i-1} \mathbf{y}
$$

Then the following hold.

(i) $\mathbf{M}$ is continuous, linear, and maximally monotone with $\operatorname{dom} \mathbf{M}=\mathbf{X}$.

(ii) $\mathbf{M}$ is rectangular.

(iii) $\operatorname{ker} \mathbf{M}=\operatorname{ker} \mathbf{M}^{*}=\boldsymbol{\Delta}$.

(iv) $\operatorname{ran} \mathbf{M}=\operatorname{ran} \mathbf{M}^{*}=\Delta^{\perp}$ is closed.

(v) $\operatorname{ran} \mathbf{L}=\Delta^{\perp}$.

(vi) $\mathbf{M} \circ \mathbf{L}=\left.\mathbf{I d}\right|_{\Delta^{\perp}}$. 
(vii) $\mathrm{M}^{-1}: \mathbf{X} \rightrightarrows \mathbf{X}: \mathbf{y} \mapsto \begin{cases}\operatorname{Ly}+\Delta, & \text { if } \mathbf{y} \in \Delta^{\perp} ; \\ \emptyset, & \text { otherwise. }\end{cases}$

(viii) $\mathbf{M}^{\dagger}=P_{\Delta^{\perp}} \circ \mathbf{L} \circ P_{\Delta^{\perp}}=\mathbf{L} \circ P_{\Delta^{\perp}}$.

(ix) $\mathbf{M}^{\dagger}=\sum_{k=1}^{m} \frac{m-(2 k-1)}{2 m} \mathbf{R}^{k-1}$.

Proof. (i): Clearly, dom $\mathbf{M}=\mathbf{X}$ and $(\forall \mathbf{x} \in \mathbf{X})\|\mathbf{R} \mathbf{x}\|=\|\mathbf{x}\|$. Thus, $\mathbf{R}$ is nonexpansive and therefore $\mathbf{M}=\mathbf{I d}-\mathbf{R}$ is maximally monotone (see, e.g., [[1], Example 20.27]).

(ii): See [[1], Example 24.14] and [[17], Step 3 in the proof of Theorem 3.1] for two different proofs of the rectangularity of $\mathbf{M}$.

(iii): The definitions of $\mathbf{M}$ and $\mathbf{R}$ and the fact that $\mathbf{R}^{*}$ is the cyclic left shift operator readily imply that ker $\mathbf{M}=\operatorname{ker} \mathbf{M}^{*}=\Delta$.

(iv), (vi), and (vii): Let $\mathbf{y}=\left(y_{1}, \ldots, y_{m}\right) \in \mathbf{X}$. Assume first that $\mathbf{y} \in$ ran $\mathbf{M}$. Then there exists $\mathbf{x}=\left(x_{1}, \ldots, x_{m}\right)$ such that $y_{1}=x_{1}-x_{m}, y_{2}=x_{2}-x_{1}, \ldots$, and $y_{m}=x_{m}-x_{m-1}$. It follows that $\sum_{i \in I} y_{i}=0$, i.e, $\mathbf{y} \in \Delta^{\perp}$ by [[1], Proposition 25.4(i)]. Thus,

$$
\operatorname{ran} \mathrm{M} \subseteq \Delta^{\perp} .
$$

Conversely, assume now that $\mathbf{y} \in \Delta^{\perp}$. Now set

$$
\mathbf{x}:=\mathbf{L y}=\sum_{i=1}^{m-1} \frac{m-i}{m} \mathbf{R}^{i-1} \mathbf{y} .
$$

It will be notationally convenient to wrap indices around, i.e., $y_{m+1}=y_{1}, y_{0}=y_{m}$ and likewise. We then get

$$
(\forall i \in I) \quad x_{i}=\frac{m-1}{m} y_{i}+\frac{m-2}{m} y_{i-1}+\cdots+\frac{1}{m} y_{i+2} .
$$

Therefore,

$$
\sum_{i \in I} x_{i}=\frac{m-1}{m} \sum_{i \in I} y_{i}+\frac{m-2}{m} \sum_{i \in I} y_{i}+\cdots+\frac{1}{m} \sum_{i \in I} y_{i}=\frac{m-1}{2} \sum_{i \in I} y_{i}=0 .
$$

Thus $\mathbf{x} \in \Delta^{\perp}$ and

$$
\operatorname{ran} \mathbf{L} \subseteq \Delta^{\perp} \text {. }
$$

Furthermore,

$$
\begin{aligned}
& (\forall i \in I) \quad x_{i}-x_{i-1}=\frac{m-1}{m} y_{i}-\frac{1}{m} y_{i-1}-\frac{1}{m} y_{i-2}-\cdots-\frac{1}{m} y_{i+1} \\
& =y_{i}-{ }_{m}^{1} \sum_{j \in I} y_{j}=y_{i} .
\end{aligned}
$$

Hence $\mathbf{M x}=\mathbf{x}-\mathbf{R x}=\mathbf{y}$ and thus $\mathbf{y} \in \operatorname{ran} \mathbf{M}$. Moreover, in view of (iii),

$$
\mathbf{M}^{-1} \mathbf{y}=\mathbf{x}+\operatorname{ker} \mathbf{M}=\mathbf{x}+\Delta .
$$

We thus have shown

$$
\boldsymbol{\Delta}^{\perp} \subseteq \operatorname{ran} \mathbf{M} .
$$


Combining (17) and (24), we obtain ran $\mathbf{M}=\Delta^{\perp}$. We thus have verified (vi), and (vii). Since ran $\mathbf{M}$ is closed, so is ran $\mathbf{M}^{*}$ (by, e.g., [[1], Corollary 15.34]). Thus (iv) holds.

(viii)\&(v): We have seen in Proposition 2.1 that

$$
\mathbf{M}^{\dagger}=P_{\text {ran }} \mathbf{M} * \circ \mathbf{M}^{-1} \circ P_{\text {ran }} \mathbf{M} .
$$

Now let $\mathbf{z} \in \mathbf{X}$. Then, by (iv), $\mathbf{y}:=P_{\text {ranM }^{z}}=P_{\Delta^{\perp z}} \in \boldsymbol{\Delta}^{\perp}$. By (vii), $\mathbf{M}^{-1} \mathbf{y}=\mathbf{L y}+\boldsymbol{\Delta}$. So $\mathbf{M}^{\dagger} \mathbf{z}=P_{\text {ran } \mathbf{M} *} \mathbf{M}^{-1} P_{\text {ran } \mathbf{M}} \mathbf{z}=P_{\text {ran } \mathbf{M}^{*}} \mathbf{M}^{-1} \mathbf{y}=P_{\mathbf{\Delta}^{\perp}}(\mathbf{L y}+\mathbf{\Delta})=P_{\mathbf{\Delta}^{\perp}} \mathbf{L y}=\mathbf{L y}=\left(\mathbf{L} \circ P_{\mathbf{\Delta}^{\perp}}\right) \mathbf{z} \quad$ because ran $\mathbf{L} \subseteq \Delta^{\perp}$ by (21). Hence (viii) holds. Furthermore, by (iv) and e.g., [[1], Proposition 3.28(v)], $\operatorname{ran} \mathbf{L}=\operatorname{ran} \mathbf{L} \circ P_{\boldsymbol{\Delta}^{\perp}}=\operatorname{ran} \mathbf{M}^{\dagger}=\operatorname{ran} \mathbf{M}^{*}=\boldsymbol{\Delta}^{\perp}$ and so (v) holds.

(ix): Note that $P_{\boldsymbol{\Delta}^{\perp}}=\mathbf{I d}-P_{\boldsymbol{\Delta}}$ and that $P_{\Delta}=m^{-1} \sum_{j \in I} \mathbf{R}^{j}$. Hence

$$
P_{\mathbf{\Delta}^{\perp}}=\mathbf{I d}-\frac{1}{m} \sum_{j \in I} \mathbf{R}^{j} .
$$

Thus, by (viii) and (16),

$$
\begin{aligned}
& \mathbf{M}^{\dagger}=\mathbf{L} \circ P_{\Delta^{\perp}}=\frac{1}{m} \sum_{i=1}^{m-1}(m-i) \mathbf{R}^{i-1} \circ\left(\mathbf{I d}-\frac{1}{m} \sum_{j \in I} \mathbf{R}^{j}\right) \\
& =\frac{1}{m} \sum_{i=1}^{m-1}(m-i) \mathbf{R}^{i-1}-\frac{1}{m^{2}} \sum_{i=1}^{m-1}(m-i) \sum_{j \in I} \mathbf{R}^{i+j-1} .
\end{aligned}
$$

Re-arranging this expression in terms of powers of $\mathbf{R}$ and simplifying leads to

$$
\mathbf{M}^{\dagger}=(\mathbf{I d}-\mathbf{R})^{\dagger}=\sum_{k=1}^{m} \frac{m-(2 k-1)}{2 m} \mathbf{R}^{k-1} .
$$

Remark 2.3 Suppose that $\tilde{\mathbf{L}}: \boldsymbol{\Delta}^{\perp} \rightarrow \mathrm{X}$ satisfies $\mathbf{M} \circ \tilde{\mathbf{L}}=\left.\mathbf{I d}\right|_{\Delta^{\perp}}$ Then

$$
\mathbf{M}^{-1}: \mathbf{X} \rightrightarrows \mathbf{X}: \mathbf{y} \mapsto \begin{cases}\widetilde{\mathbf{L}} y+\Delta, & \text { if } \mathbf{y} \in \Delta^{\perp} \\ \emptyset, & \text { otherwise }\end{cases}
$$

One may show that $\mathbf{M}^{\dagger}=P_{\Delta^{\perp}} \circ \tilde{\mathbf{L}} \circ P_{\Delta^{\perp}}$ and that $P_{\Delta^{\perp}} \circ \tilde{\mathbf{L}}=\mathbf{L}$ (see (16)). Concrete choices for $\tilde{\mathbf{L}}$ and $\mathbf{L}$ are

$$
\boldsymbol{\Delta}^{\perp} \rightarrow \mathbf{X}:\left(y_{1}, y_{2}, \ldots, y_{m}\right) \mapsto\left(y_{1}, y_{1}+y_{2}, \ldots, y_{1}+y_{2}+y_{3}+\ldots+y_{m}\right) ;
$$

however, the range of the latter operator is not equal $\Delta^{\perp}$ whenever $X \neq\{0\}$.

Remark 2.4 Denoting the symmetric part of $\mathbf{M}$ by $\mathbf{M}_{+}=\frac{1}{2} \mathbf{M}+\frac{1}{2} \mathbf{M}^{*}$ and defining the quadratic form associated with $\mathbf{M}$ by $q_{\mathrm{M}}: \mathrm{x} \rightarrow \frac{1}{2}\langle\mathrm{x}, \mathrm{Mx}\rangle$, we note that [[17], Proposition 2.3] implies that ${ }^{\mathrm{c}}$ ran $\mathbf{M}_{+}=\operatorname{dom} q_{\mathrm{M}}^{*}=\boldsymbol{\Delta}^{\perp}$.

Fact 2.5 (Brezis-Haraux) (See [20] and also, e.g., [[1], Theorem 24.20].) Suppose $A$ and $B$ are monotone operators on $X$ such that $A+B$ is maximally monotone, $\operatorname{dom} A \subseteq$ $\operatorname{dom} B$, and $B$ is rectangular. Then int $\operatorname{ran}(A+B)=\operatorname{int}(\operatorname{ran} A+\operatorname{ran} B)$ and $\overline{\operatorname{ran}(A+B)}=\overline{\operatorname{ran} A+\operatorname{ran} B}$. 
Applying the Brezis-Haraux result to our given operators $\mathbf{A}$ and $\mathbf{M}$, we obtain the following.

Corollary 2.6 The operator $\mathbf{A}+\mathbf{M}$ is maximally monotone and $\overline{\operatorname{ran}(\mathbf{A}+\mathbf{M})}=\overline{\mathbf{\Delta}^{\perp}+\operatorname{ran} \mathbf{A}}$

Proof. Since each $A_{i}$ is maximally monotone and recalling Theorem 2.2(i), we see that $\mathbf{A}$ and $\mathbf{M}$ are maximally monotone. On the other hand, $\operatorname{dom} \mathbf{M}=\mathbf{X}$. Thus, by the well known sum theorem for maximally monotone operators (see, e.g., [[1], Corollary 24.4 (i)]), $\mathbf{A}+\mathbf{M}$ is maximally monotone. Furthermore, by Theorem 2.2(ii) and (iv), $\mathbf{M}$ is rectangular and ran $\mathbf{M}=\Delta^{\perp}$. The result therefore follows from Fact 2.5.

\section{Composition}

We now use Corollary 2.6 to study the composition. When $m=2$, then Theorem 3.1 (v) also follows from [[21], p. 124].

Theorem 3.1 Suppose that $(\forall i \in I) 0 \in \overline{\operatorname{ran}\left(\mathrm{Id}-T_{i}\right)}$. Then the following hold.

(i) $\mathbf{0} \in \overline{\operatorname{ran}(\mathrm{A}+\mathrm{M})}$.

(ii) $(\forall \varepsilon>0)(\exists(\mathbf{b}, \mathbf{x}) \in \mathbf{X} \times \mathbf{X})\|\mathbf{b}\| \leq \varepsilon$ and $\mathbf{x}=\mathbf{T}(\mathbf{b}+\mathbf{R x})$.

(iii) $(\forall \varepsilon>0)(\exists(\mathbf{c}, \mathbf{x}) \in \mathbf{X} \times \mathbf{X})\|\mathbf{c}\| \leq \varepsilon$ and $\mathbf{x}=\mathbf{c}+\mathbf{T}(\mathbf{R x})$.

(iv) $(\forall \varepsilon>0)(\exists \mathbf{x} \in \mathbf{X})(\forall i \in I)\left\|T_{i-1} \cdots T_{1} x_{m}-T_{i} T_{i-1} \cdots T_{1} x_{m}-x_{i-1}+x_{i}\right\| \leq(2 i-$

1) $\varepsilon$, where $x_{0}=x_{m}$.

(v) $(\forall \varepsilon>0)(\exists x \in X)\left\|x-T_{m} T_{m-1} \cdots T_{1} x\right\| \leq m^{2} \varepsilon$.

Proof. (i): The assumptions and (3) imply that $(\forall i \in I) 0 \in \overline{\operatorname{ran} A_{i}}$. Hence, $\mathbf{0} \in \overline{\operatorname{ran} \mathbf{A}}$. Obviously, $\mathbf{0} \in \Delta^{\perp}$. It follows that $\mathbf{0} \in \overline{\boldsymbol{\Delta}^{\perp}+\operatorname{ran} \mathbf{A}}$. Thus, by Corollary 2.6, $0 \in \overline{\operatorname{ran}(\mathrm{A}+\mathrm{M})}$.

(ii): Fix $\varepsilon>0$. In view of (i), there exists $\mathbf{x} \in \mathbf{X}$ and $\mathbf{b} \in \mathbf{X}$ such that $\|\mathbf{b}\| \leq \varepsilon$ and $\mathbf{b}$ $\in \mathbf{A x}+\mathbf{M x}$. Hence $\mathbf{b}+\mathbf{R} \mathbf{x} \in(\mathbf{I d}+\mathbf{A}) \mathbf{x}$ and thus $\mathbf{x}=J_{\mathbf{A}}(\mathbf{b}+\mathbf{R x})=\mathbf{T}(\mathbf{b}+\mathbf{R x})$.

(iii): Let $\varepsilon>0$. By (ii), there exists $(\mathbf{b}, \mathbf{x}) \in \mathbf{X} \times \mathbf{X}$ such that $\|\mathbf{b}\| \leq \varepsilon$ and $\mathbf{x}=\mathbf{T}(\mathbf{b}+$ $\mathbf{R x})$. Set $\mathbf{c}=\mathbf{x}-\mathbf{T}(\mathbf{R} \mathbf{x})=\mathbf{T}(\mathbf{b}+\mathbf{R} \mathbf{x})-\mathbf{T}(\mathbf{R x})$. Then, since $\mathbf{T}$ is nonexpansive, $\|\mathbf{c}\|=$ $\|\mathbf{T}(\mathbf{b}+\mathbf{R x})-\mathbf{T}(\mathbf{R} \mathbf{x})\| \leq\|\mathbf{b}\| \leq \varepsilon$.

(iv): Take $\varepsilon>0$. Then, by (iii), there exists $\mathbf{x} \in \mathbf{X}$ and $\mathbf{c} \in \mathbf{X}$ such that $\|\mathbf{c}\| \leq \varepsilon$ and $\mathbf{x}=\mathbf{c}+\mathbf{T}(\mathbf{R x})$. Let $i \in I$. Then $x_{i}=c_{i}+T_{i} x_{i-1}$. Since $\left\|c_{i}\right\| \leq\|\mathbf{c}\| \leq \varepsilon$ and $T_{i}$ is nonexpansive, we have

$$
\begin{aligned}
& \left\|T_{i} T_{i-1} \cdots T_{1} x_{0}-x_{i}\right\| \leq\left\|T_{i} T_{i-1} \cdots T_{1} x_{0}-T_{i} x_{i-1}\right\|+\left\|T_{i} x_{i-1}-x_{i}\right\| \\
& \leq\left\|T_{i} T_{i-1} \cdots T_{1} x_{0}-T_{i} x_{i-1}\right\|+\varepsilon
\end{aligned}
$$

We thus obtain inductively

$$
\left\|T_{i} T_{i-1} \cdots T_{1} x_{0}-x_{i}\right\| \leq i \varepsilon .
$$

Hence,

$$
\left\|T_{i-1} \cdots T_{1} x_{0}-x_{i-1}\right\| \leq(i-1) \varepsilon .
$$


The conclusion now follows from adding (33) and (34), and recalling the triangle inequality

(v): Let $\varepsilon>0$. In view of (iv), there exists $\mathbf{x} \in \mathbf{X}$ such that

$$
(\forall i \in I) \quad\left\|T_{i-1} \cdots T_{1} x_{m}-T_{i} T_{i-1} \cdots T_{1} x_{m}-x_{i-1}+x_{i}\right\| \leq(2 i-1) \varepsilon
$$

where $x_{0}=x_{m}$. Now set $(\forall i \in I) e_{i}=T_{i-1} \cdots T_{1} x_{m}-T_{i} T_{i-1} \cdots T_{1} x_{m}-x_{i-1}+x_{i}$. Then $(\forall i \in I)\left\|e_{i}\right\| \leq(2 i-1) \varepsilon$. Set $x=x_{m}$. Then

$$
\begin{aligned}
& \sum_{i=1}^{m} e_{i}=\sum_{i=1}^{m} T_{i-1} \ldots T_{1} x_{m}-T_{i} T_{i-1} \ldots T_{1} x_{m}-x_{i-1}+x_{i} \\
& =x-T_{m} T_{m-1} \ldots T_{1} x .
\end{aligned}
$$

This, (35), and the triangle inequality imply that

$$
\left\|x-T_{m} T_{m-1} \cdots T_{1} x\right\| \leq \sum_{i=1}^{m}\left\|e_{i}\right\| \leq \sum_{i=1}^{m}(2 i-1) \varepsilon=m^{2} \varepsilon .
$$

This completes the proof.

Corollary $\quad 3.2 \quad$ Suppose that $\quad(\forall i \in I) 0 \in \overline{\operatorname{ran}\left(\operatorname{Id}-T_{i}\right)}$. Then $0 \in \overline{\operatorname{ran}\left(\mathrm{Id}-T_{m} T_{m-1} \cdots T_{1}\right)}$.

Proof. This follows from Theorem 3.1(v).

Remark 3.3 The converse implication in Corollary 3.2 fails in general: indeed, consider the case when $X \neq\{0\}, m=2$, and $v \in X \backslash\{0\}$. Now set $T_{1} X \rightarrow X: x \mapsto x+v$ and set $T_{2} X \rightarrow X: x \mapsto x-v$. Then $0 \notin \overline{\operatorname{ran}\left(\operatorname{Id}-T_{1}\right)}=\{-v\}$ and $0 \notin \overline{\operatorname{ran}\left(\operatorname{Id}-T_{2}\right)}=\{v\}$ however, $T_{2} T_{1}=\mathrm{Id}$ and $\overline{\operatorname{ran}\left(\mathrm{Id}-T_{2} T_{1}\right)}=\{0\}$.

Remark 3.4 Corollary 3.2 is optimal in the sense that even if $(\forall i \in I)$ we have $0 \in$ $\operatorname{ran}\left(\mathrm{Id}-T_{i}\right)$, we cannot deduce that $0 \in \operatorname{ran}\left(\mathrm{Id}-T_{m} T_{m-1} \cdots T_{1}\right)$ : indeed, suppose that $X=\mathbb{R}^{2}$ and $m=2$. Set $C_{1}:=$ epi exp and $C_{2}:=\mathbb{R} \times\{0\}$. Suppose further that $T_{1}=P_{C_{1}}$ and $T_{2}=P_{C_{2}}$. Then $(\forall i \in I) \quad 0 \in \operatorname{ran}\left(\mathrm{Id}-T_{i}\right)$; however, $0 \in \overline{\operatorname{ran}\left(\mathrm{Id}-T_{2} T_{1}\right)} \backslash \operatorname{ran}\left(\mathrm{Id}-T_{2} T_{1}\right)$.

\section{Asymptotic regularity}

The following notions (taken from Bruck and Reich's seminal article [22]) will be very useful to obtain stronger results.

Definition 4.1 ((strong) nonexpansiveness and asymptotic regularity) Let $S: X \rightarrow$ $X$. Then:

(i) $S$ is nonexpansive if $(\forall x \in X)(\forall y \in X)\|S x-S y\| \leq\|x-y\|$.

(ii) $S$ is strongly nonexpansive if $S$ is nonexpansive and whenever $\left(x_{n}\right)_{n \in \mathbb{N}}$ and $\left(y_{n}\right)$ $n \in \mathbb{N}$ are sequences in $X$ such that $\left(x_{n}-y_{n}\right)_{n \in \mathbb{N}}$ is bounded and $\left\|x_{n}-y_{n}\right\|-\| S x_{n}$ $S y_{n} \| \rightarrow 0$, it follows that $\left(x_{n}-y_{n}\right)-\left(S x_{n}-S y_{n}\right) \rightarrow 0$.

(iii) $S$ is asymptotically regular if $(\forall x \in X) S^{n} x-S^{n+1} x \rightarrow 0$.

The following result illustrates that strongly nonexpansive mappings generalize the notion of a firmly nonexpansive mapping. In addition, the class of strongly 
nonexpansive mappings is closed under compositions. (In contrast, the composition of two (necessarily firmly nonexpansive) projectors may fail to be firmly nonexpansive.)

Fact 4.2 (Bruck and Reich) The following hold.

(i) Every firmly nonexpansive mapping is strongly nonexpansive.

(ii) The composition of finitely many strongly nonexpansive mappings is also strongly nonexpansive.

Proof. (i): See [[22], Proposition 2.1]. (ii): See [[22], Proposition 1.1].

The sequences of iterates and of differences of iterates have striking convergence properties as we shall see now. In passing, we note that Fact 4.3(i) also appears in [[21], Theorem 3.7(b)] even in certain Banach spaces.

Fact 4.3 (Bruck and Reich) Let $S: X \rightarrow X$ be strongly nonexpansive and let $x \in X$. Then the following hold.

(i) The sequence $\left(S^{n} x-S^{n+1} x\right)_{n \in \mathbb{N}}$ converges strongly to the unique element of least norm in $\overline{\operatorname{ran}(\mathrm{Id}-S)}$.

(ii) If Fix $S=\varnothing$, then $\left\|S^{n} x\right\| \rightarrow+\infty$.

(iii) If Fix $S \neq \varnothing$, then $\left(S^{n} x\right)_{n \in \mathbb{N}}$ converges weakly to a fixed point of $S$.

Proof (i): See [[22], Corollary 1.5]. (ii): See [[22], Corollary 1.4]. (iii): See [[22], Corollary 1.3].

Suppose $S: X \rightarrow X$ is asymptotically regular. Then, for every $x \in X, 0 \Re S^{n} x-S^{n+1} x$ $=(\operatorname{Id}-S) S^{n} x \in \operatorname{ran}(\operatorname{Id}-S)$ and hence $0 \in \overline{\operatorname{ran}(\operatorname{Id}-S)}$. The opposite implication fails in general (consider $S=-$ Id), but it is true for strongly nonexpansive mappings. Under the assumption that $S$ is firmly nonexpansive, the following result also follows from [[23], Corollary 2].

Corollary 4.4 Let $S: X \rightarrow X$ be strongly nonexpansive. Then $S$ is asymptotically regular if and only if $0 \in \overline{\operatorname{ran}(\mathrm{Id}-S)}$.

Proof. " $\Rightarrow$ ": Clear. " $\Leftarrow$ ": Fact 4.3(i).

Corollary 4.5 Set $S=T_{m} T_{m}-1 \cdots T_{1}$. Then $S$ is asymptotically regular if and only if $0 \in \overline{\operatorname{ran}(\mathrm{Id}-S)}$.

Proof Since each $T_{i}$ is firmly nonexpansive, it is also strongly nonexpansiveby Fact 4.2 (i). By Fact 4.2(ii), $S$ is strongly nonexpansive. Now apply Corollary 4.4. Alternatively, $0 \in \overline{\operatorname{ran}(\mathrm{Id}-S)}$ by Corollary 3.2 and again Corollary 4.4 applies.

We are now ready for our first main result. When $m=2$, then the conclusion also follows from [[21], p. 124].

Theorem 4.6 Suppose that each $T_{i}$ is asymptotically regular. Then $T_{m} T_{m-1} \cdots T_{1}$ is asymptotically regular as well.

Proof. Theorem 3.1(v) implies that $0 \in \overline{\operatorname{ran}\left(\mathrm{Id}-T_{m} T_{m-1} \cdots T_{1}\right)}$. The conclusion thus follows from Corollary 4.5.

As an application of Theorem 4.6, we obtain the main result of [17].

Example 4.7 Let $C_{1}, \ldots, C_{m}$ be nonempty closed convex subsets of $X$. Then the composition of the corresponding projectors, $P_{C_{m}} P_{C_{m-1}} \ldots P_{C_{1}}$ is asymptotically regular. 
Proof. For every $i \in I$, the projector $P_{C_{i}}$ is firmly nonexpansive, hence strongly nonexpansive, and Fix $P_{C_{i}}=C_{i} \neq \emptyset$. Suppose that $(\forall i \in I) T_{i}=P_{C_{i}}$, which is thus asymptotically regular by Corollary 4.4. Now apply Theorem 4.6.

\section{Convex combination}

In this section, we use our fixed weights $\left(\lambda_{i}\right)_{i \in I}$ (see (12)) to turn $X^{m}$ into a Hilbert product space different from $\mathbf{X}$ considered in the previous sections. Specifically, we set

$$
\mathbf{Y}:=X^{m} \quad \text { with } \quad\langle\mathbf{x}, \mathbf{y}\rangle=\sum_{i \in I} \lambda_{i}\left\langle x_{i}, y_{i}\right\rangle
$$

so that $\|\mathbf{x}\|^{2}=\sum_{i \in I} \lambda_{i}\left\|x_{i}\right\|^{2}$. We also set

$$
\text { Q: } X^{m} \rightarrow X^{m}: \mathbf{x} \mapsto(\bar{x}) i \in I, \quad \text { where } \bar{x}:=\sum_{i \in I} \lambda_{i} x_{i} .
$$

Fact 5.1 (See [[1], Proposition 28.13].) In the Hilbert product space $\mathbf{Y}$ we have $P_{\Delta}=$ Q.

Corollary 5.2 In the Hilbert product space $\mathbf{Y}$ the operator $\mathbf{Q}$ is firmly nonexpansive and strongly nonexpansive. Furthermore, Fix $\mathbf{Q}=\Delta \neq \varnothing, \mathbf{0} \in \operatorname{ran}(\mathbf{I d}-\mathbf{Q})$, and $\mathbf{Q}$ is asymptotically regular.

Proof By Fact 5.1, the operator $\mathbf{Q}$ is equal to the projector $P_{\Delta}$ and hence firmly nonexpansive. Now apply Fact 4.2(i) to deduce that $\mathbf{Q}$ is strongly nonexpansive. It is clear that Fix $\mathbf{Q}=\Delta$ and that $\mathbf{0} \in \operatorname{ran}(\mathbf{I d}-\mathbf{Q})$. Finally, recall Corollary 4.4 to see that $\mathbf{Q}$ is asymptotically regular.

Proposition 5.3 In the Hilbert product space $\mathbf{Y}$ the operator $\mathbf{T}$ is firmly nonexpansive.

Proof. Since each $T_{i}$ is firmly nonexpansive, we have $\left(\forall \mathbf{x}=\left(x_{i}\right)_{i \in I} \in \mathbf{Y}\right)\left(\forall \mathbf{y}=\left(y_{i}\right)_{i \in I} \in\right.$ Y) $\left\|T_{i} x_{i}-T_{i} y_{i}\right\|^{2} \leq\left\langle x_{i}-y_{i}, T_{i} x_{i}-T_{i} y_{i}\right\rangle \Rightarrow\|\mathbf{T} \mathbf{x}-\mathbf{T} \mathbf{y}\|^{2}=\sum_{i \in I} \lambda_{i}\left\|T_{i} x_{i}-T_{i} y_{i}\right\|^{2} \leq \sum_{i \in I} \lambda_{i}\left\langle x_{i}\right.$ $\left.-y_{i}, T_{i} x_{i}-T_{i} y_{i}\right\rangle=\langle\mathbf{x}-\mathbf{y}, \mathbf{T} \mathbf{x}-\mathbf{T} \mathbf{y}\rangle$.

Theorem 5.4 Suppose that $(\forall i \in I) 0 \in \overline{\operatorname{ran}\left(\mathrm{Id}-T_{i}\right)}$. Then the following hold in the Hilbert product space $\mathbf{Y}$.

(i) $0 \in \overline{\operatorname{ran}(\mathbf{I d}-\mathrm{T})}$.

(ii) $\mathbf{T}$ is asymptotically regular.

(iii) $\mathbf{Q} \circ \mathbf{T}$ is asymptotically regular.

Proof. (i): This follows because $\left(\forall \mathbf{x}=\left(x_{i}\right)_{i \in I}\right)\|\mathbf{x}-\mathbf{T} \mathbf{x}\|^{2}=\sum_{i \in I} \lambda_{i}\left\|x_{i}-T_{i} x_{i}\right\|^{2}$.

(ii): Combine Fact 4.2(i) with Corollary 4.4.

(iii): On the one hand, $\mathbf{Q}$ is firmly nonexpansive and asymptotically regular by Corollary 5.2. On the other hand, $\mathbf{T}$ is firmly nonexpansive and asymptotically regular by Proposition 5.3 and Theorem 5.4(ii). Altogether, the result follows from Theorem 4.6.

We are now ready for our second main result, which concerns convex combinations of firmly nonexpansive mappings. For further results in this direction-namely convex 
combinations of strongly nonexpansive mappings in Banach spaces-we refer the reader also to [24].

Theorem 5.5 Suppose that each $T_{i}$ is asymptotically regular. Then $\sum_{i \in I} \lambda_{i} T_{i}$ is asymptotically regular as well.

Proof. Set $S:=\sum_{i \in I} \lambda_{i} T_{i}$. Fix $x_{0} \in X$ and set $(\forall n \in \mathbb{N}) x_{n+1}=S x_{n}$. Set $\mathbf{x}_{0}=\left(x_{0}\right)_{i \in I} \in$ $X^{m}$ and $(\forall n \in \mathbb{N}) \mathbf{x}_{n+1}=(\mathbf{Q} \circ \mathbf{T}) \mathbf{x}_{n}$. Then $(\forall n \in \mathbb{N}) \mathbf{x}_{n}=\left(x_{n}\right)_{i \in I}$. Now $\mathbf{Q} \circ \mathbf{T}$ is asymptotically regular by Theorem 5.4(iii); hence, $\mathbf{x}_{n}-\mathbf{x}_{n+1}=\left(x_{n}-x_{n+1}\right)_{i \in I} \rightarrow \mathbf{0}$. Thus, $x_{n}-x_{n+1} \rightarrow 0$ and therefore $S$ is asymptotically regular.

Remark 5.6 Theorem 5.5 extends [[18], Theorem 4.11] from Euclidean to Hilbert space. One may also prove Theorem 5.5 along the lines of the article [18]; however, that route takes longer.

Remark 5.7 Similarly to Remark 3.4, one cannot deduce that if each $T_{i}$ has fixed points, then $\sum_{i \in I} \lambda_{i} T_{i}$ has fixed points as well: indeed, consider the setting described in Remark 3.4 for an example.

We conclude this article by showing that we truly had to work in $\mathbf{Y}$ and not in $\mathbf{X}$; indeed, viewed in $\mathbf{X}$, the operator $\mathbf{Q}$ is generally not even nonexpansive.

Theorem 5.8 Suppose that $X \neq\{0\}$. Then the following are equivalent in the Hilbert product space $\mathbf{X}$.

(i) $(\forall i \in I) \lambda_{i}=1 / m$.

(ii) $\mathbf{Q}$ coincides with the projector $P_{\Delta}$.

(iii) $\mathbf{Q}$ is firmly nonexpansive.

(iv) $\mathbf{Q}$ is nonexpansive.

Proof. "(i) $\Rightarrow($ ii)": [[1], Proposition 25.4(iii)]. “(ii) $\Rightarrow($ iii)": Clear. “(iii) $\Rightarrow($ iv)": Clear. "(iv) $\Rightarrow(\mathrm{i}) "$ ": Take $e \in X$ such that $\|e\|=1$. Set $\mathbf{x}:=\left(\lambda_{i} e\right)_{i L I}$ and $y:=\sum_{i \in I} \lambda_{i}^{2} e$. Then $\mathbf{Q x}=$ $(y)_{i \in I}$. We compute $\|\mathbf{Q} \mathbf{x}\|^{2}=m\|y\|^{2}=m\left(\sum_{i \in I} \lambda_{i}^{2}\right)^{2}$ and $\|\mathbf{x}\|^{2}=\sum_{i \in I} \lambda_{i}^{2}$. Since $\mathbf{Q}$ is nonexpansive, we must have that $\|\mathbf{Q x}\|^{2} \leq\|\mathbf{x}\|^{2}$, which is equivalent to

$$
m\left(\sum_{i \in I} \lambda_{i}^{2}\right)^{2} \leq \sum_{i \in I} \lambda_{i}^{2}
$$

and to

$$
m \sum_{i \in I} \lambda_{i}^{2} \leq 1
$$

On the other hand, applying the Cauchy-Schwarz inequality to the vectors $\left(\lambda_{i}\right)_{i \in I}$ and $(1)_{i \in I}$ in $\mathbb{R}^{m}$ yields

$$
1=1^{2}=\left(\sum_{i \in I} \lambda_{i} \cdot 1\right)^{2} \leq\left\|\left(\lambda_{i}\right)_{i \in I}\right\|^{2}\left\|(1)_{i \in I}\right\|^{2}=m \sum_{i \in I} \lambda_{i}^{2} .
$$

In view of (42), the Cauchy-Schwarz inequality (43) is actually an equality which implies that $\left(\lambda_{i}\right)_{i \in I}$ is a multiple of $(1)_{i \in I}$. We deduce that $(\forall i \in I) \lambda_{i}=1 / \mathrm{m}$.

\section{Endnotes}

${ }^{\mathrm{a}} \operatorname{ker} B=B^{-1} 0=\{x \in X \mid B x=0\}$ denotes the kernel (or nullspace) of $B$. 
${ }^{\text {b}}$ Here and elsewhere we write $S^{n}$ for the $n$-fold composition of an operator $S$. ${ }^{\mathrm{c}}$ Recall that the Fenchel conjugate of a function $f$ defined on $X$ is given by $f^{*}: x^{*} \rightarrow$ $\sup _{x \in X}\left(\left\langle x, x^{*}\right\rangle-f(x)\right)$.

\section{Acknowledgements}

The authors thank Simeon Reich, the editor, and the referees for constructive and pertinent comments. Part of this research was initiated during a research visit of VM-M at the Kelowna campus of UBC in Fall 2009. HHB was partially supported by the Natural Sciences and Engineering Research Council of Canada and by the Canada Research Chair Program. VM-M was partially supported by DGES, Grant BFM2009-1096-C02-01 and Junta de Andalucia, Grant FQM127. SMM was partially supported by the Natural Sciences and Engineering Research Council of Canada. XW was partially supported by the Natural Sciences and Engineering Research Council of Canada.

\section{Author details}

'Mathematics, University of British Columbia, Kelowna, BC V1V 1V7, Canada ${ }^{2}$ Departamento de Análisis Matemático, Universidad de Sevilla, Apdo. 1160, 41080-Sevilla, Spain

\section{Authors' contributions}

All authors contributed equally to this research. All authors read and approved the final manuscript.

\section{Competing interests}

The authors declare that they have no competing interests.

Received: 22 December 2011 Accepted: 26 March 2012 Published: 26 March 2012

\section{References}

1. Bauschke, HH, Combettes, PL: Convex Analysis and Monotone Operator Theory in Hilbert Spaces. Springer, New York (2011)

2. Goebel, K, Kirk, WA: Topics in Metric Fixed Point Theory. Cambridge University Press, Cambridge (1990)

3. Goebel, K, Reich, S: Uniform Convexity, Hyperbolic Geometry, and Nonexpansive Mappings. Marcel Dekker, New York (1984)

4. Minty, GJ: Monotone (nonlinear) operators in Hilbert spaces. Duke Math J. 29, 341-346 (1962). doi:10.1215/S0012-709462-02933-2

5. Eckstein, J, Bertsekas, DP: On the Douglas-Rachford splitting method and the proximal point algorithm for maximal monotone operators. Math Program (Ser A). 55, $293-318$ (1992). doi:10.1007/BF01581204

6. Borwein, JM, Vanderwerff, JD: Convex Functions. Cambridge University Press, Cambridge (2010)

7. Brézis, H: Operateurs Maximaux Monotones et Semi-Groupes de Contractions dans les Espaces de Hilbert. NorthHolland/Elsevier, New York (1973)

8. Burachik, RS, lusem, AN: Set-Valued Mappings and Enlargements of Monotone Operators. Springer-Verlag, New York (2008)

9. Rockafellar, RT: Convex Analysis. Princeton University Press, Princeton (1970)

10. Rockafellar, RT, Wets, RJ-B: Variational Analysis, corrected 3rd printing. Springer-Verlag, Berlin (2009)

11. Simons, S: Minimax and Monotonicity. Springer-Verlag, Berlin (1998)

12. Simons, S: From Hahn-Banach to Monotonicity. Springer-Verlag, New York (2008)

13. Zălinescu, C: Convex Analysis in General Vector Spaces. World Scientific Publishing, River Edge, NJ (2002)

14. Zeidler, E: Nonlinear Functional Analysis and Its Applications II/A: Linear Monotone Operators. Springer-Verlag, New York (1990)

15. Zeidler, E: Nonlinear Functional Analysis and Its Applications II/B: Nonlinear Monotone Operators. Springer-Verlag, New York (1990)

16. Zeidler, E: Nonlinear Functional Analysis and Its Applications I: Fixed Point Theorems. Springer-Verlag, New York (1993)

17. Bauschke, $\mathrm{HH}$ : The composition of finitely many projections onto closed convex sets in Hilbert space is asymptotically regular. Proc Am Math Soc. 131, 141-146 (2003). doi:10.1090/S0002-9939-02-06528-0

18. Bauschke, HH, Moffat, SM, Wang, X: Near equality, near convexity, sums of maximally monotone operators, and averages of firmly nonexpansive mappings. Mathematical Programming (in press). http://arxiv.org/pdf/1105.0029v1

19. Groetsch, CW: Generalized Inverses of Linear Operators. Marcel Dekker, New York (1977)

20. Brézis, H, Haraux, A: Image d'une somme d'opérateurs monotones et applications. Israel J Math. 23, 165-186 (1976). doi:10.1007/BF02756796

21. Reich, S: On the asymptotic behavior of nonlinear semigroups and the range of accretive operators. J Math Anal Appl. 79, 113-126 (1981). doi:10.1016/0022-247X(81)90013-5

22. Bruck, RE, Reich, S: Nonexpansive projections and resolvents of accretive operators in Ba-nach spaces. Houston J Math. 3, 459-470 (1977)

23. Reich, S, Shafrir, I: The asymptotic behavior of firmly nonexpansive mappings. Proc Am Math Soc. 101, $246-250$ (1987). doi:10.1090/S0002-9939-1987-0902536-7

24. Reich, S: A limit theorem for projections. Linear and Multilinear Algebra. 13, 281-290 (1983). doi:10.1080/ 03081088308817526

doi:10.1186/1687-1812-2012-53

Cite this article as: Bauschke et al:: Compositions and convex combinations of asymptotically regular firmly nonexpansive mappings are also asymptotically regular. Fixed Point Theory and Applications 2012 2012:53. 